\title{
ATHENS SEASONAL VARIATION OF GROUND RESISTANCE PREDICTION USING NEURAL NETWORKS
}

\author{
S. Anbazhagan \\ Department of Electrical Engineering, Annamalai University, India \\ E-mail: s.anbazhagan@gmx.com
}

\begin{abstract}
The objective in ground resistance is to attain the most minimal ground safety esteem conceivable that bodes well monetarily and physically. An application of artificial neural networks (ANN) to presage and relegation has been growing rapidly due to sundry unique characteristics of ANN models. A decent forecast is able to capture the dubiousness associated with those ground resistance. A portion of the key instabilities are soil composition, moisture content, temperature, ground electrodes and spacing of the electrodes. Propelled by this need, this paper endeavors to develop a generalized regression neural network (GRNN) to predict the ground resistance. The GRNN has a single design parameter and expeditious learning and efficacious modeling for nonlinear time series. The precision of the forecast is applied to the Athens seasonal variation of ground resistance that shows the efficacy of the proposed approach.
\end{abstract}

Keywords:

Ground Resistance, Generalized Regression Neural Network, Forecasting

\section{INTRODUCTION}

Equipment's failure and downtime are owing to poor grounding. Good grounding is indispensable otherwise it leads an electric shock, instrumentation errors, harmonic distortion and power factor issues. It needs zero resistance path to ground when fault current occurs if it finds unintended paths that include human being.

Every year people lose their lives due to electrical fires. Due to this consequence, zero resistance paths are indispensable for the dissipation of fault currents, lightning strikes, static discharges, electromagnetic interference and radio frequency interference. The following four methods are available for earth ground testing: (1) Soil resistivity, (2) Fall-of-potential, (3) Selective and (4) Stakeless quantification. In this study, subsisting quantification values of Athens soil resistivity and ground resistance are acclimated to presage the future values utilizing neural networks.

Determining the design of good grounding system for installing new equipments depends on soil resistivity. The soil resistivity depends on composition, moisture content and temperature of soils. Soil is heterogeneous in nature and resistivity of the soil will vary place to place and at different soil depths. Soil resistivity measurements are often corrupted by the existence of ground currents and their harmonics. The soil resistivity and ground resistance quantifying procedure described in [1-3] utilizes the macrocosmically accepted Wenner method developed by Dr. Frank Wenner.

The statistical quantifications of subsisting grounding system arrangements are discussed in [4-9]. In these studies, the influence on the demeanor of grounding electrodes soil layer and its resistivity has been investigated. These techniques are habituated to quantify the grounding resistance predicated on electrode and soil parameters.

Another consequential research effort is on the development of an artificial neural network (ANN) approach to supersede the current statistical methods. To that end, M.A. Salam et al [10] proposed an ANN approach is utilized to model and presage the relationship between the grounding resistance and the length of the buried electrode in the soil predicated on experimental data. F.E. Asimakopoulou et al [1-3] have presaged the demeanor of the ground resistance of a single rod during a period of time by utilizing ANN that have been trained with experimental data of soil resistivity and weather conditions. On that purport a methodology for the optimization of the parameters of different training algorithms and the cull of the optimum algorithm has been implemented. Their works, however, was inhibited to multilayer perceptron networks (MLP) only and their methods showed lowering performance in the prediction. MLP networks are flexible computing frameworks for modeling a broad range of nonlinear time series. Realizing that MLP networks have several shortcomings, it suffers from a slow convergence rate and often yields suboptimal solutions.

Hence, in this paper, the generalized regression neural network (GRNN) model is to predict the Athens experimental data of soil resistivity and ground resistance is proposed. GRNN has only a single design parameter and is simple and in turn it trains the network.

The rest of the paper is organized as follows. Section 2 outlines the proposed methodology for forecasting seasonal variation of ground resistance. Section 3 presents the numerical results of proposed GRNN model simulations. Finally, conclusions are described in Section 4.

\section{METHODOLOGY}

This section of the research describes the experimental data, GRNN model for forecasting seasonal variation of ground resistance and their prediction results analysis.

\subsection{EXPERIMENTAL DATA}

The precision of a prediction model significantly depends on the precision of the data source. In this study, 67 (training and evaluation set) as the training dataset, and 10 (test set) for testing. Both datasets were anteriorly published in F.E. Asimakopoulou et al [1-3] and also used as standard benchmark data.

Feature cull can be defined as a quandary of finding a set of minimum number of germane features that describes the dataset. In the dataset, all samples have 5 input features comprises the apparent soil resistivity quantifications (in $\Omega \mathrm{m}$ ) for electrode 
distances at $1 \mathrm{~m}$ and $2 \mathrm{~m}$, the average rainfall during the preceding week, the rainfall during the day on which the ground resistance is estimated (in $\mathrm{mm}$ ) and the average resistance during the preceding week (in $\Omega$ ). The output feature is the ground resistance (in $\Omega$ ). The soil resistivity and ground resistance quantifications of the soil resistivity were conducted in the area of Athens [1-3].

\subsection{GRNN GROUND RESISTANCE PREDICTION MODEL}

The ANNs are vigorous implements for the presage and simulation in sundry engineering applications. In this study, GRNN is culled among the main neural network architecture utilized in seasonal variation of ground resistance due to single design parameter and expeditious learning and efficacious modeling for non linear time series. Therefore a GRNN model is developed with anterior day rainfall, antecedent week rainfall, soil resistivity quantifications for electrode at distance $1 \mathrm{~m}, 2 \mathrm{~m}$ and precedent week resistance as inputs and ground resistance as desired output.

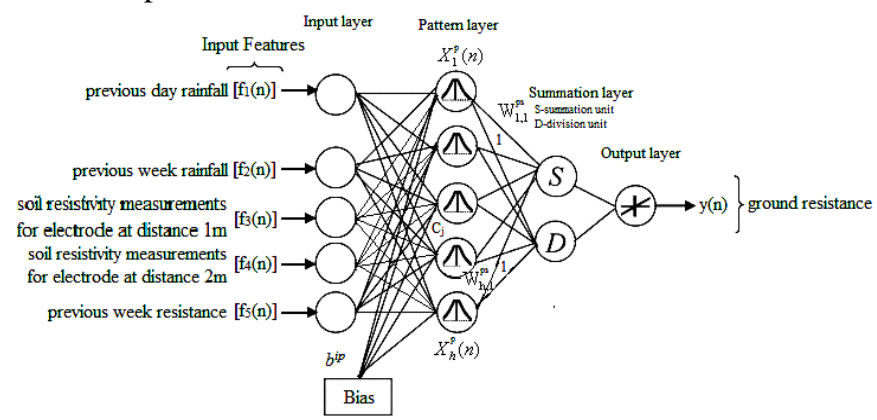

Fig.1. Implementation of GRNN for the seasonal variation of ground resistance forecasting

The GRNN [11-13] is a feed forward neural network predicated on non-linear regression theory consisting of four layers, the input layer, the pattern layer, the summation layer, and the output layer. The architecture of GRNN is shown in Fig. 1 to forecast the seasonal variation of ground resistance.

$$
\begin{gathered}
\vec{X}_{j}^{p}=\phi\left(\left\|\vec{f}-\vec{c}_{j}\right\| \times \vec{b}^{i p}\right) \\
\phi(x)=\mathrm{e}^{-x 2} \\
\vec{b}^{i p}=\frac{0.8326}{s} \\
y=\frac{\sum_{j=1}^{h} \vec{W}_{j}^{p s} \times \vec{X}_{j}^{p}}{\sum_{j=1}^{h} \vec{X}_{j}^{p}}
\end{gathered}
$$

where, $\vec{f}(n)$ values are the input features, $j=1,2, \ldots, h$ (number of hidden neurons), $\vec{X}_{j}^{p}(n)$ value is the $j^{\text {th }}$ output of pattern layer, $\vec{W}^{p s}(n)$ are the weights from the pattern layer to summation layer, $\vec{c}_{j}$ is the center vector of the $j^{\text {th }}$ node, $s$ is the real constant known as spread factor, $\vec{b}^{i p}(n)$ is the biasing term of pattern layer, $\phi($.$) is the non-linear radial basis function (Gaussian), y(n)$ value is the output for forecast ground resistance and $n$ is training pattern index.

The neurons in the input, pattern and summation layers are connected fully but output layer neurons are connected only to some processing units in the summation layer. The pattern layer neurons are computing their transfer function by radial basis function, which is typically the Gaussian kernel function. If feed zero value to radial basis function, it gives maximum of one value. Hence, the distance between the input vector and the pattern unit weight vector decreases, then output of pattern units are increased. Thus the pattern layer neurons act as a detector which produces maximum of value one whenever the input vector is identical to its pattern unit weight vector. One of the parameters for GRNN is the spread of radial basis functions. The spread default value is 1. The more astronomically immense the spread the smoother is the function approximation. To fit the data proximately, one can utilize a spread more diminutive than the typical distance between the input vectors. To fit the data more smoothly a more immensely colossal spread can be utilized.

The summation layer has two variants of neural processing the summation neuron units and a single neuron division unit. The number of neurons in the summation layer is always equal to the number of the GRNN output neurons. The single neuron division unit is weighted activation of pattern neurons without utilizing any transfer function.

The training procedure of the GRNN and the back propagation neural network are entirely different. Firstly, it calculates the weight vector for each input-output vector pair from the training set for pattern layer only once. Finally, it computes the distance between the new input vector and pattern unit weight vector as computed early; and the processing units in the summation layer are assigned simultaneously. The GRNN computes the soothsaid values 'on the fly' from the training values, utilizing the radial basis functions. The calculation of output activation procedure is follows:

1. The activation of input units is resolute by the example presented to the network.

2. The activation of pattern unit is given in Eq.(1).

3. The activation of each summation unit is a linear sum of weighted inputs from the pattern layer.

4. The activation of the output unit (Eq.(4)) is the activation of summation unit divided by that of division unit.

\subsection{ANALYSIS OF THE RESULTS}

The input features and the target output are linearly normalized in the range of $\{-1,1\}$ afore being presented to the GRNN model. Then, the output from the GRNN model was denormalized afore being presented in the presage performance evaluation. The min-max method is utilized to normalize and denormalize the features are described in $[14,15]$. The performance of the GRNN network was then evaluated by comparison of the network output with its actual value via statistical evaluation. The Mean Absolute Percentage Error (MAPE), Mean Absolute Deviation (MAD) and Mean Squared Deviation (MSD) are utilized to evaluate the performance of prediction in ground resistance.

The MAPE can be defined as, 


$$
M A P E=\frac{1}{N} \sum_{i=1}^{N}\left|\frac{A_{i}-F_{i}}{A_{i}}\right| \times 100
$$

The MAD is given by,

$$
M A D=\frac{1}{N} \sum_{i=1}^{N}\left|A_{i}-F_{i}\right|
$$

The MSD is given by,

$$
M S D=\frac{1}{N} \sum_{i=1}^{N}\left|A_{i}-F_{i}\right|^{2}
$$

where, $A_{i}$ and $F_{i}$ are the actual and predicted value of the ground resistance of $i^{\text {th }}$ data, respectively and $N$ is the number of forecasted data.

The MAPE is one of the commonly used measures of prediction precision, which quantifications the proportionality between the authentic and prognosticated ground resistance. MAD helps conceptualize the amount of error. MSD used measure of accuracy of fitted time series values. Hence, the data replication in the experiment is represented by evaluation of the resulting GRNN performance in terms of MAPE.

\section{NUMERICAL RESULTS}

This section describes the testing of Athens seasonal variation of ground resistance prediction by the proposed GRNN approach and comparison results with ANN model.

\subsection{GROUND RESISTANCE PREDICTION WITH GRNN MODEL}

In the GRNN architecture, the spread factor was resolute utilizing trial and error approach. An illimitable number of endeavors were made until the best spread factor was engendered minimal error in both training and testing.

The proposed GRNN model has the input layer composed of 5 neurons. The size of the input vector is 5 (previous day rainfall, previous week rainfall, soil resistivity measurements for electrode at distance $1 \mathrm{~m}, 2 \mathrm{~m}$ and previous week resistance) $\times 67$ (training and evaluation set) and the size of the target vector is 1 (ground resistance) $\times 67$. This configuration is proven to be a macrocosmic mapping, provided that the best spread factor values. It should be noted that each of the testing case studies was run many times to evaluate different spread factor in the model. The GRNN prediction models are implemented utilizing the Matrix Laboratory (MATLAB) neural network toolbox.

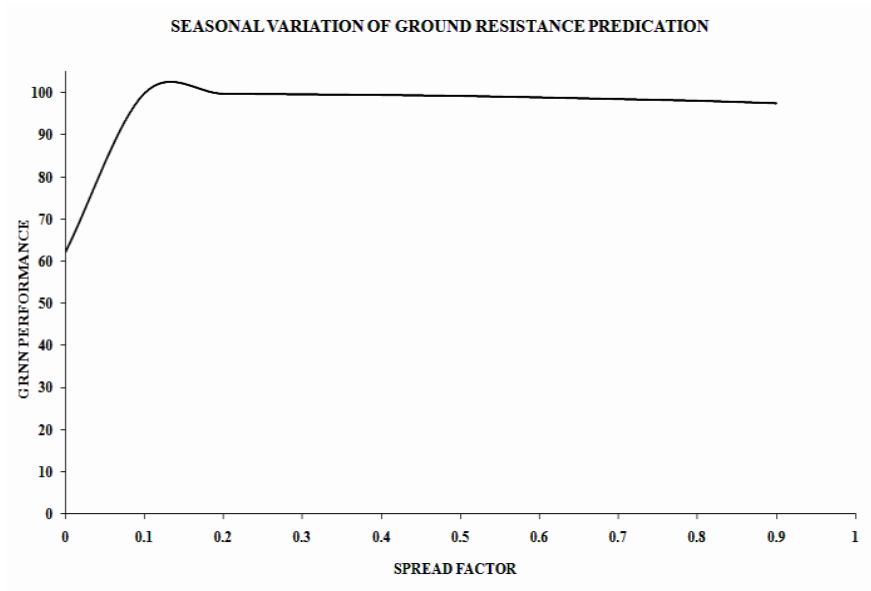

Fig.2. Performance curve of GRNN for seasonal variation of ground resistance

The Fig. 2 shows the spread factor versus the performance soothsaid by GRNN for seasonal variation of ground resistance. The performance of GRNN increases for the spread factor values from 0.1 to 0.2 is shown in Fig. 2. Maximum performance $99.91 \%$ occurs at the spread value 0.1355 . After that the performance of GRNN is gradually reducing for seasonal variation of ground resistance. The best spread factor value 0.1355 for GRNN prediction model produced minimal in both training and testing.

\subsection{COMPARISON OF PREDICTION RESULTS WITH ANN MODEL}

The Table.1 summarizes the numerical results, where the comparison of prediction performance of the proposed GRNN and ANN is presented. The first column denotes the quantified/actual ground resistance, the second betokens the ANN estimated ground resistance, the third denotes the proposed GRNN estimated ground resistance and the last row shows the MAPE. It is observed that the MAPE, MAD and MSD for the proposed GRNN model have an average value of $0.940 \%, 0.200$ and 0.110 respectively. From the same table the MAPE, MAD and MSD for the ANN model has an average value of $1.776 \%$, 0.350 and 0.229 respectively.

Amelioration in the average MAPE of the proposed GRNN model with veneration to the ANN model is 47.07\%. An ANN model has 7000 epochs and longer computational time. But, the total setup time of the GRNN model including the execution of normalization, testing of GRNN and de-normalization was about less than $1 \mathrm{~s}$ on an AMD processor with $2 \mathrm{GHz}$ clock speed and 1GB RAM memory. After training, average computation time of the GRNN was about less than $15 \mathrm{~ms}$ (since it only involves with the forward propagation of the GRNN). So, we can facilely verbally express that the proposed GRNN has good prediction precision with less computation time. Hence, it is opportune for the real-time. 
Table.1. Comparative MAPE results between the proposed GRNN estimated values and ANN models

\begin{tabular}{|c|c|c|}
\hline $\begin{array}{c}\text { Measured / Actual } \\
\text { Ground Resistance }\end{array}$ & $\begin{array}{c}\text { Estimated by } \\
\text { ANN[3] }\end{array}$ & $\begin{array}{c}\text { Estimated by } \\
\text { GRNN }\end{array}$ \\
\hline 34.9 & 34.8 & 34.9 \\
\hline 38.0 & 38.4 & 38.2 \\
\hline 19.3 & 18.8 & 19.4 \\
\hline 19.5 & 19.2 & 19.5 \\
\hline 20.0 & 19.6 & 19.6 \\
\hline 27.8 & 27.8 & 27.6 \\
\hline 16.7 & 16.8 & 16.7 \\
\hline 18.2 & 18.1 & 18.2 \\
\hline 19.5 & 19.9 & 19.7 \\
\hline 18.8 & 20.0 & 19.7 \\
\hline
\end{tabular}

\begin{tabular}{|c|c|c|}
\hline MAPE & 1.776 & 0.940 \\
\hline MAD & 0.350 & 0.200 \\
\hline MSD & 0.229 & 0.110 \\
\hline
\end{tabular}

\section{CONCLUSION}

A precise prediction of ground resistance is consequential for industrial plants and equipment. In this paper, a GRNN has been proposed for forecasting seasonal variation of ground resistance. Prediction results of Athens seasonal variation of ground resistance, yielding an average MAPE is proximate to $0.940 \%$, while the average computation time is less than $15 \mathrm{~ms}$. The GRNN model has only a single design parameter and is simple and in turn it trains the network. The simulation results from the comparisons limpidly show that the GRNN model is good in prediction precision than the ANN prediction. The research work is underway in order to develop better forecast models for quantified seasonal variation of ground resistance.

\section{REFERENCES}

[1] F.E. Asimakopoulou, E.A. Kourni, V.T. Kontargyri, G.J. Tsekouras and I.A. Stathopulos, "Artificial Neural Network Methodology for the Estimation of Ground Resistance", Proceedings of WSEAS International Conference on Systems, pp. 453-458, 2011.

[2] F.E. Asimakopoulou, G.J. Tsekouras, I.F. Gonos and I.A. Stathopulos, "Artificial Neural Network Approach on the Seasonal Variation of Soil Resistance", Proceedings of $7^{\text {th }}$ Asia-Pacific International Conference on Lightning, pp. 794-799, 2011.

[3] F.E. Asimakopoulou, G.J. Tsekouras, I.F. Gonos and I.A. Stathopulos, "Estimation of Seasonal Variation of Ground Resistance using Artificial Neural Networks", Electric Power Systems Research, Vol. 94, pp. 113-121, 2013.
[4] Robert L. Cascio, "Safe Measurement of Ground Resistance", Proceedings of Society for Mining, Metallurgy and Exploration Symposium, 1992.

[5] T. Takahashi and T. Kawase, "Calculation of Earth Resistance for a Deep Driven Rod in a Multi-layer Earth Structure", IEEE Transactions on Power Delivery, Vol. 6, No. 2, pp. 608-614, 1991.

[6] C.J. Blattner, "Prediction of Soil Resistivity and Ground Rod Resistance for Deep Ground Electrode," IEEE Transactions on Power Apparatus and Systems, Vol. PAS99, No. 5, pp. 1758-1763, 1980.

[7] Y.L. Chow and M.M.A. Salama, "A Simplified Method for Calculating the Substation Grounding Grid Resistance," IEEE Transactions on Power Delivery, Vol. 9 No. 2, pp. 736-742, 1994.

[8] F. Dawalibi and N. Barbetio, "Measurements and Computations of the Performance of Grounding System Buried in Multilayer Soils", IEEE Transactions on Power Delivery, Vol. 6 No. 4, pp. 1483-1490, 1991.

[9] M.A. Salam, A.A. Maqrashi, N. Mohammed, Z. Nadir and M. Shahidullah, "Statistical Approach to Find an Empirical Relationship between the Grounding Resistance and Length of Buried Electrode in the Soil", WSEAS Transactions on Circuits and Systems, Vol. 3, No. 6, pp. 1483-1486, 2004.

[10] M.A. Salam, S.M. Al-Alawi and A.A. Maqrashi, "An Artificial Neural Networks Approach to Model and Predict the Relationship between the Grounding Resistance and Length of Buried Electrode in the Soil", Journal of Electrostatics, Vol. 64, No. 5, pp. 338-342, 2006.

[11] D.F. Specht, "A General Regression Neural Network", IEEE Transactions on Neural Networks, Vol. 2, No. 6, pp. 568$576,1991$.

[12] S. Anbazhagan and N. Kumarappan, "Day-ahead Deregulated Electricity Market Price Forecasting using Neural Network Input Featured by DCT", Energy Conversion and Management, Vol. 78, pp. 711-719, 2014.

[13] S. Anbazhagan and N. Kumarappan, "Day-ahead Deregulated Electricity Market Price Forecasting using Recurrent Neural Network", IEEE Systems Journal, Vol. 7, No. 4, pp. 866-872, 2013.

[14] S. Anbazhagan and N. Kumarappan, "Day-ahead Price Forecasting in Asia's First Liberalized Electricity Market using Artificial Neural Networks", International Journal of Computational Intelligence Systems, Vol. 4, No. 4, pp. 476$485,2011$.

[15] MATLAB Help, “General Regression Neural Network". Available at: http://in.mathworks.com/help/nnet/ug/generalizedregression-neural-networks.html. Accessed 05 October 2015. 